\title{
Inolvidables con Lucho Gatica (1958): un tributo jazzístico a la canción mexicana ${ }^{1}$ Inolvidables con Lucho Gatica (1958): A Jazz Tribute to Mexican Song
}

\author{
por \\ Daniel Party \\ Instituto de Música, Facultad de Artes \\ Pontificia Universidad Católica de Chile, Chile \\ dparty@uc.cl
}

Este artículo busca poner en valor el elepé Inolvidables con Lucho (Odeón, 1958), del cantante chileno Lucho Gatica, mediante tres líneas argumentales. Primero, Inolvidables con Lucho es uno de los primeros elepés latinoamericanos de música popular unificados en cuanto a repertorio (canción romántica mexicana de la primera mitad del siglo veinte) y acompañamiento instrumental (un ensamble pequeño de jazz liderado por el guitarrista mexicano Arturo Castro). Como tal, demuestra una concepción del formato elepé similar a la establecida por Frank Sinatra y Capitol Records. Segundo, el disco adopta una práctica asociada a cantantes mujeres estadounidenses de grabar repertorio standard con acompañamiento de guitarra jazz y contrabajo. En tercer lugar, se considera el impacto sustancial, pero poco reconocido, que Inolvidables con Lucho tuvo entre los músicos que desarrollaron la bossa nova en Brasil a fines de la década de 1950.

Palabras clave: Elepé, música mexicana, jazz, bossa nova.

This article seeks to assess the importance of Chilean singer Lucho Gatica's long playing record Inolvidables con Lucho (Odeón, 1958). It proposes three lines of argument. First, Inolvidables constitutes one of the first Latin American LP albums unified in terms of repertoire-Mexican 'canción romántica' from the first half of the twentieth century-, and instrumental accompaniment - a small jazz ensemble featuring Mexican guitarist Arturo Castro-. As such, it demonstrates an understanding of the LP format in line with the one established by Frank Sinatra and Capitol Records. Second, the album adopts a practice associated with US women singers of recording standards accompanied by jazz guitar and bass. Third, the substantial, but under-recognized impact Inolvidables con Lucho had on the artists who developed bossa nova in Brazil in the late 1950s is considered.

Keywords: LP, Mexican music, jazz, bossa nova.

1 Este trabajo forma parte del Proyecto FONDECYT Regular No 1140979 "Listening to Gender: A Hermeneutics of Music as Performance", cuyo investigador responsable es el autor de este artículo. Agradezco el aporte esencial que realizó el guitarrista de jazz Sebastián Prado, quien en calidad de personal técnico del proyecto realizó análisis musical y transcripciones. Versiones preliminares de esta investigación fueron presentadas en Tulane University (Gilbert Chase Memorial Annual Lecture in Latin American Music, 18/10/2012) y en el II Simposio Jazz en América Latina, en el marco del XI Congreso de la Rama Latinoamericana de la IASPM (Salvador, Brasil, 14/10/2014). 
En la segunda mitad de la década de 1950, el cantante chileno Lucho Gatica (n. 1928) se encontraba en la cima de la fama. Radicado en México desde 1955, sus grabaciones de boleros como "Encadenados" y "La Barca", con acompañamiento de la orquesta de José Sabre Marroquín, eran éxito en toda América Latina. En México compartía escenarios y la pantalla del cine con artistas como Miguel Aceves Mejía y Pedro Vargas. En La Habana, el exótico "Lucho" era admirado por sus elegantes interpretaciones del repertorio cubano; en Perú, su memorable paso por Lima quedó para siempre novelado por Mario Vargas Llosa en La tía Julia y el escribidor (1977); y en Brasil era reconocido como "uno de los artistas más apreciados de América Latina, especialmente por las jóvenes" ${ }^{2}$. En Estados Unidos la disquera Capitol Records lo eligió como su principal apuesta para lanzar al mercado norteamericano una nueva serie de elepés ${ }^{3}$ con repertorio extranjero -un intento temprano de establecer una suerte de world music. Titulada "Capitol of the World", la serie incluyó varios álbumes de Gatica con nombres de fácil pronunciación, como Mucho Lucho, Lara by Lucho y El Gran Gatica ${ }^{4}$.

En medio de esta vorágine, Gatica grabó para la disquera Odeón un elepé titulado Inolvidables con Lucho $(1958)^{5}$. A pesar del poco afortunado nombre, que sugiere otra colección más de grandes éxitos, Inolvidables es un álbum profundamente innovador y distinto, tanto de lo que Gatica venía haciendo hasta ese momento como de lo que grabó posteriormente. Inspirado por el potencial que ofrecía el formato de doce pulgadas, Inolvidables es un ejemplo temprano de un álbum de música popular latinoamericana unificado en cuanto a repertorio -canciones románticas mexicanas de la primera mitad del siglo veinte-, y en cuanto a acompañamiento instrumental -solo una guitarra y un contrabajo en la mayoría de las canciones, guitarra, bajo y bongó en las otras.

En Inolvidables acompañan a Gatica dos músicos mexicanos, Arturo Castro en guitarra y Víctor Ruiz Pazos en contrabajo, a los que se suma un bongocero no identificado. Castro era un joven prodigio que comenzó su carrera junto con sus hermanos en Los Panchitos, un trío romántico que imitaba a la perfección a Los Panchos ${ }^{6}$. En los años sesenta el trío se expande y transforma en Los Hermanos Castro, un cuarteto vocal que alcanzó gran éxito en México y Estados Unidos. Además, Castro es compositor, particularmente recordado por canciones como "Llorando por dentro", "Y después del amor" y "Yo sin ti". Pazos, por su parte, es un destacado contrabajista veracruzano de jazz, con amplia experiencia en big bands.

2 Long Playing, III/16, p. 98. Todas las traducciones son del autor.

3 Utilizo el sustantivo masculino elepé en vez de la expresión inglesa long play, la sigla LP o el calco larga duración siguiendo la recomendación realizada por la RAE en 2005.

4 Para un estudio del estilo de Gatica y su recepción, ver Party 2012. Existen dos biografías disponibles de Gatica, Rojas Donoso 1992 y Márquez 2013.

5 El disco fue editado por Odeón en distintos países, con nombres ligeramente distintos: Inolvidables con Lucho en Cuba, Chile y México; Boleros inolvidables con Lucho en Argentina; Inolvidables con Lucho Gatica en Brasil.

6 Se puede ver a Arturo Castro, de unos 10 años, tocando requinto y cantando junto con sus hermanos en la película Arrabalera (1950). 
Desde el primer acorde de Inolvidables con Lucho, una tónica con sexta y novena, es evidente que el estilo con que Castro y Ruiz Pazos acompañan a Gatica no es lo que se esperaba en la época para canciones mexicanas antiguas. Castro toca una guitarra con cuerdas de nylon, pero no a la manera de los tríos románticos, sino en el estilo de guitarristas de jazz de la posguerra, como Barney Kessel o Mundell Lowe. Esto resulta audible en el uso de acordes extendidos, sustituciones y voicings de jazz ${ }^{7}$.

El objetivo de este artículo es poner en valor este elepé relativamente desconocido en la discografía de Lucho Gatica. Para este fin propongo tres líneas argumentales. Primero, Inolvidables con Lucho es uno de los primeros elepés latinoamericanos de música popular unificados en cuanto a repertorio, ensamble y estilo musical. Como tal, demuestra una concepción del formato elepé similar a la establecida por Frank Sinatra y Capitol Records. Segundo, el disco adopta una práctica asociada a cantantes mujeres estadounidenses de grabar repertorio standard con acompañamiento de guitarra jazz y contrabajo. En tercer lugar, considero el impacto sustancial, pero poco reconocido, que Inolvidables con Lucho tuvo entre los músicos que desarrollaron la bossa nova en Brasil a fines de la década de 1950 .

\section{EL ELEPÉ, CAPITOL Y SINATRA}

La introducción del disco de "Larga Duración" de 33 RPM -el de 10 pulgadas en 1948 y el de 12 en 1954- tuvo un impacto profundo en la producción y el consumo musicales. Como sugiere el nombre, la característica más importante del nuevo formato era que permitía un tiempo de reproducción considerablemente más largo que los tres minutos y medio de los antiguos discos de 78 RPM. También importante es el hecho que el vinilo, el material con que se fabricaban los elepés, era más durable que el shellac de los viejos discos de 78 RPM. Por esta razón, una de las estrategias comerciales al momento de introducir los discos elepé fue destacar su permanencia e indestructibilidad.

En su historia del vinilo, Osborne documenta que el elepé fue diseñado para la reproducción de música clásica, en parte porque obras extensas y unificadas ya existían, en parte porque el repertorio clásico ya contaba con un canon de obras consideradas dignas de conservación y permanencia ${ }^{8}$. Y en un principio el público estuvo de acuerdo: a comienzos de los años cincuenta, los elepés más

7 En Cuba existía, desde mediados de los cuarenta, un estilo de interpretar canciones influenciado por el jazz norteamericano, y que se conoce como filin, del inglés feeling (Contreras 1989; Giro 2001; García Yero 2012). Varios de los músicos fundadores de este estilo eran guitarristas-compositores, como César Portillo de la Luz y José Antonio Méndez, que en presentaciones en vivo solían acompañarse a sí mismos en un estilo sofisticado de guitarra jazz. Para grabaciones comerciales, sin embargo, en los cuarenta y cincuenta se consideraba que el acompañamiento con guitarra no era de gusto masivo. En palabras de Guillermo Rodríguez Rivera, "El filin, música para voz y guitarra, si quería acceder a los grandes medios, a la radio, la televisión, el disco, tenía que acondicionarse a la estandarización, plegarse a orquestaciones que vendieran" (Contreras 1989: 81).

8 Osborne 2012: 87-115. 
vendidos en Estados Unidos no eran de cantantes populares o de jazz, sino de música clásica ${ }^{9}$.

En el ámbito de la música popular, el elepé contribuyó al establecimiento de una división de repertorios por formato. Canciones nuevas destinadas al hit parade continuaron siendo distribuidas en discos sencillos de 78 RPM -luego reemplazados por sencillos de 45 RPM-. Los elepés de música popular, por su parte, consistían principalmente en "canciones de catálogo" o standards. En esta división de repertorios por formato también subyacía una segregación de mercado, con los sencillos destinados a audiencias jóvenes y los elepés a consumo adulto. En 1952, el vicepresidente de Capitol Records declaró: "El desarrollo futuro de la industria discográfica depende no tanto de los adolescentes y los compradores de discos sencillos de pop sino en otro mercado con gustos completamente distintos: personas jóvenes casadas y personas mayores, que son importantes para la venta de álbumes"10. Osborne explica esta segregación etaria haciendo referencia al hecho que, para los adolescentes, el elepé era demasiado caro y menos atractivo, ya que requería un cuidado especial por su fragilidad ${ }^{11}$.

El músico popular que logró capitalizar de forma más productiva la división de mercado entre sencillos y elepés fue Frank Sinatra, a partir de 1953, en sus grabaciones para Capitol Records ${ }^{12}$. Como resume Keightley,

Sus elepés para Capitol (exceptuando sus compilaciones de grandes éxitos) contenían standards de pop adulto, mientras que sus discos sencillos buscaban el éxito efímero del Top 40 con canciones compuestas recientemente. En sus 14 álbumes originales temáticos o "conceptuales" para Capitol no hay canciones que fueran éxitos de los ránkings, y sus elepés de la década del cincuenta lograron codificar un repertorio específico de standards de pop para adultos ${ }^{13}$.

La adopción que hizo Sinatra del formato elepé y de un repertorio standard es parte de una reinvención de su carrera, con el objetivo de alejarse de las fans adolescentes y entrar en un mercado adulto. Roger Gilbert describe la transformación de Sinatra como un proceso en el que "el crooner frágil, tenue y juvenil que hacía que las calcetineras mojaran los asientos del Teatro Paramount se transformara en el amante desenfrenado, duro, sofisticado, con el sombrero hacia adelante, un cigarrillo colgando y chaqueta sobre el hombro" 14 .

Sinatra y Capitol también fueron pioneros en la producción de elepés unificados temáticamente ${ }^{15}$. Esta unificación se extiende al contenido musical, mediante el uso de un mismo grupo de músicos y un solo arreglista por disco, y al diseño de la carátula. Como observa Osborne, Sinatra simultáneamente aprovechó y

\footnotetext{
9 Keightley 2008:319.

10 Keightley 2004: 375.

11 Osborne 2012: 94.

12 Osborne 2012: 100.

13 Keightley 2001: 29.

14 Gilbert 1998:41.

15 Osborne 2012: 98-100.
} 
potenció las asociaciones que ya existían en torno al elepé: "serio, adulto, refinado, 'no comercial', masculino (en el sentido de que el mundo de la alta fidelidad era considerado como dominado por hombres)" 16 . El resultado, al menos en términos de popularidad, es indiscutible: de todas las ventas de elepés en el período 195659, incluida la música clásica, el artista más exitoso era Frank Sinatra, seguido por Johnny Mathis ${ }^{17}$.

\section{LUCHO GATICA CANTA EL CANCIONERO ROMÁNTICO MEXICANO}

Como la mayoría de los cantantes populares de los cincuenta, Gatica construyó su fama con discos sencillos de 78 RPM, no álbumes de larga duración. En la segunda mitad de la década sus primeros elepés de 10 y 12 pulgadas consistían en material grabado con anterioridad, en general una mezcla de éxitos recientes y material de catálogo. Estas colecciones no eran particularmente satisfactorias, ya que presentaban una yuxtaposición poco orgánica de repertorios, arreglos y acompañamientos. Inolvidables con Lucho rompe esta tradición al ser un disco concebido como un elepé unificado. Siguiendo la práctica de la industria norteamericana de poblar los elepés con standards, Inolvidables no incluye canciones nuevas, sino solo clásicos. Esto en sí ya era una apuesta para Gatica, quien, desde su llegada a México en 1955, había alcanzado la fama privilegiando canciones compuestas poco tiempo antes y de autores jóvenes como Álvaro Carrillo (1921-1969), Roberto Cantoral (1935-2010) y José Antonio Méndez (1927-1989).

El repertorio de Inolvidables consiste en un cancionero (songbook) de canción romántica mexicana compuesta durante la primera mitad del siglo veinte. "Canción romántica” es un concepto amplio que define un carácter y no un género o forma musical. Es significativo que incluso Carlos Monsiváis, el autor más elocuente que ha escrito acerca de la música romántica mexicana, se vio forzado a formular una generalidad al tratar de definirla: "el término canción romántica corresponde más a una definición cultural y literaria que a un estado de ánimo"18. Canción romántica ha incluido una variedad de estilos musicales, de modo particular la canción mexicana en la década de 1910 y el bolero después de la mitad de la década del veinte, pero también la habanera, el vals, el beguine y el arrullo ${ }^{19}$ (ver Cuadro 1).

16 Osborne 2012: 99.

17 Stephens 2008:173.

18 Monsiváis 1984: 24.

19 Monsiváis 1984: 24 
CUADRO 1

LISTA DE CANCIONES DEL DISCO INOLVIDABLES CON LUCHO, CON SUS AUTORES Y AÑOS DE COMPOSICIÓN

\begin{tabular}{|l|l|c|}
\hline \multicolumn{2}{|c|}{ Nombre canción } & \multicolumn{2}{|c|}{ Autores } & $\begin{array}{c}\text { Año de } \\
\text { composición }\end{array}$ \\
\hline Cara A & Agustín Lara & 1935 \\
\hline Noche de ronda & Gonzalo Curiel & 1935 \\
\hline Tú & Ignacio Fernández Esperón & 1917 \\
\hline La borrachita & José Sabre Marroquín/José Mojica & 1937 \\
\hline Nocturnal & Manuel María Ponce & 1913 \\
\hline A la orilla de un palmar & Mario Ruiz Armengol/Fernando Soto & 1944 \\
\hline Por qué llorar & Gabriel Ruiz/Ricardo López Méndez & 1941 \\
\hline Noche & & 1936 \\
\hline Cara B & Alberto Domínguez & 1939 \\
\hline Frenesí & Miguel Prado Paz/Gabriel Luna de la Fuente & 1936 \\
\hline Duerme & Ricardo López Méndez/Guty Cárdenas & 1935 \\
\hline Nunca & María Grever & 1938 \\
\hline Alma mía & Mario Talavera/Rubén C. Navarro & \\
\hline Déjame llorar & & \\
\hline Arrullo & & \\
\hline
\end{tabular}

En Inolvidables están representadas tres generaciones de autores mexicanos, de manera proporcional, aunque no cronológica. La canción más antigua, "A la orilla de un palmar", es una adaptación del folklore realizada en 1913 por el compositor clásico Manuel M. Ponce. En la década de 1910 Ponce comenzó un proyecto de recopilación y arreglo de canciones folklóricas tanto para piano solo como para voz y acompañamiento de piano, que denominó "canciones mexicanas". El objetivo de Ponce, argumenta la musicóloga Leonora Saavedra, era "pulir" 
canciones vernáculas para convertirlas en arte elevado para las clases educadas medias y altas $^{20}$.

En ensayos y conferencias, Ponce elaboró respecto de la importancia del "folklore" mexicano y su potencial uso para la música clásica, y su proyecto terminó teniendo un impacto mucho más amplio que lo que él imaginó cuando lo comenzó ${ }^{21}$. A fines de la década de 1910 Ponce se había convertido en "el padre de la música vernácula mexicana”, y un grupo de compositores urbanos, algunos sin formación de conservatorio, continuaron su propuesta, "produciendo una profusión de arreglos y canciones originales que fueron publicadas en las mismas casas editoriales en las que Ponce publicaba"22. Inolvidables reconoce a esta generación al incluir canciones de sus compositores más relevantes, Ignacio Fernández Esperón (1894-1968), Mario Talavera (1885-1960), Alfonso Esparza Oteo (1898-1950) y María Grever (1885-1951). Característico de las letras de este primer periodo es la construcción nostálgica de lo rural desde el espacio urbano, ejemplificado en Inolvidables en "La borrachita" y "A la orilla de un palmar".

La siguiente generación de compositores, nacidos en la primera década del siglo, es la más representada en Inolvidables con Lucho. Agustín Lara (1900-1970), Gonzalo Curiel (1904-1958), Agustín ‘Guty’ Cárdenas (1905-1932), Miguel Prado Paz (1905-1987) y José Sabre Marroquín (1909-1995), fueron los primeros autores en capitalizar el surgimiento de la industria cultural mexicana. Durante la década de 1930, la radio, el cine y las grabaciones le dieron a estos compositores una presencia mediática desconocida hasta ese momento, tanto a nivel nacional como internacional ${ }^{23}$. También durante esa década el bolero se convierte en el estilo más popular de la canción romántica mexicana. Los primeros éxitos de esta generación de compositores marcan el comienzo de lo que se conoce como la era dorada del bolero mexicano, que se extiende hasta fines de los años cincuenta, cuando se graba Inolvidables. A diferencia del interés en lo rural, de la generación anterior, para esta generación el foco es la urbe, en particular los espacios citadinos, bohemios y nocturnos -lo que está ilustrado en este disco con "Noche de ronda" y "Nocturnal".

La generación más joven de compositores representados en Inolvidables nació en la década de 1910, e incluye a Gabriel Ruiz (1912-1999), Alberto Domínguez (1913-1975), Mario Ruiz Armengol (1914-2002) y Consuelo Velázquez (19162005). Monsiváis caracteriza a esta generación como "profesionales de la música" en el sentido que, a diferencia de Lara, ellos "piensan y deliberan sus carreras, las conciben en términos fundamentalmente económicos y publicitarios" 24 . Estos autores se mantienen en un segundo plano cuando sus canciones se convierten en éxitos en la década de 1940, en grabaciones, ya no de tenores con formación clásica, sino de crooners cuya voz es amplificada electrónicamente.

\footnotetext{
20 Saavedra 2009: 284.

21 Saavedra 2009: 285.

22 Saavedra 2009: 285.

23 Monsiváis 1984: 35.

24 Monsiváis 1984:31.
} 
Desde Odeón, la compañía discográfica de Gatica, tenían claro que el foco en repertorio antiguo era una característica esencial del disco -ahí radica el título del elepé-. La edición cubana del álbum, realizada por Odeón-Panart, omite mencionar la nacionalidad de los autores, pero destaca que "famosos compositores crearon estas canciones inolvidables... que han perdurado a través de todos los tiempos”25. La contratapa de la edición chilena sí reconoce el origen mexicano del repertorio (la edición brasileña incluye el mismo texto, pero en traducción al portugués), y lo presenta como un homenaje al país al que Gatica emigró en 1955. "Lucho Gatica con este disco agradece a México y a los autores mexicanos el estímulo de su aplauso cordial y sincero" y las canciones son "joyas líricas que, creadas por los más brillantes compositores, representan más de medio siglo de fructífera labor musical" -aunque, en efecto, solo 35 años separan a la canción más antigua de la más reciente-.

Es posible observar la misma claridad en la recepción desde la prensa especializada. En el número de enero-febrero de 1959, la revista brasileña Long Playing, revista bimestral abocada a las novedades de la industria del disco, dedica un párrafo a promocionar el lanzamiento de Inolvidables con Lucho. Long Playing presenta a Gatica como un cantante especialmente apreciado por "las jóvenes" de América Latina, gracias a su estilo "caliente (quente)", al "calor con que dice las palabras de las melodías románticas" 26 . En cuanto al contenido de este nuevo disco, la revista se centra en que "Gatica procura revivir páginas conocidísimas", lo que es luego enfatizado, "un elepé en que procura revivir viejas canciones mexicanas" 27 .

Aunque ni las carátulas ni la prensa lo reconocen, el repertorio de Inolvidables con Lucho está también unificado en cuanto al carácter nocturno, íntimo y melancólico de las canciones. Poe escribe que "el drama de la relación amorosa que escenifica el bolero se actualiza en un espacio nocturno, bohemio, transgresor" 28 . Cuatro canciones del disco refieren de manera explícita a estos espacios nocturnos, "Nocturnal", "Noche", "Duerme" y "Arrullo". Pero más que bohemio, Inolvidables releva la noche como un espacio íntimo, tierno y privado. En todo el disco, Gatica rara vez canta con gran volumen, y en la cara B del disco pareciera que el objetivo es arrullar al auditor, gracias a delicadas canciones de cuna como "Duerme" y "Arrullo". La tristeza, por su parte, es especialmente evidente en la recurrencia con que aparece el llanto y el llorar ("Por qué llorar" y "Déjame llorar", "A la orilla de un palmar" y "Noche de ronda").

25 Debido a la conocida resistencia hacia el bolero mexicano por parte de las clases medias y altas cubanas, no es sorprendente que esta edición no hace ninguna referencia al hecho que todos los compositores y músicos acompañantes eran mexicanos (ver Acosta 1988).

26 Long Playing, III/16: 98.

27 Long Playing, III/16: 98-100.

28 Poe 1996: 19. 


\section{CON ARREGLO ESPECIAL: CROONERSY JAZZ}

Inolvidables es un disco unificado no solo por su repertorio, sino también por el uso de un mismo ensamble en todo el disco. Hasta donde he podido constatar, el ensamble voz - guitarra jazz - bajo no tiene precedentes en la discografía de la canción romántica mexicana con anterioridad a Inolvidables con Lucho. Sin embargo, en el mundo de los crooners estadounidenses estaba en pleno proceso una renovación de los grupos acompañantes. A causa de la cercanía que Gatica tenía con el mundo anglosajón durante los años cincuenta, desde su paso por Capitol Records hasta su colaboración con el arreglista Nelson Riddle, es poco probable que el cantante no estuviera al tanto de dicha tendencia.

A partir de mediados de la década de los cincuenta, destacados crooners norteamericanos grabaron con grupos pequeños, en reemplazo de orquestas o big bands. En su estudio acerca de los crooners, Stephens identifica que grabar con ensambles de este tipo es otra manera de unificar un elepé, una alternativa a la unificación por repertorio ${ }^{29}$. En virtud que la grabación con orquestas era el estándar para los crooners, discos como estos eran llamados "con arreglo especial (special arrangement album)". Entre los discos "con arreglo especial” se encuentran grabaciones de cantantes con trío de jazz (piano, contrabajo, batería), con piano solo, o con una o dos guitarras jazz y contrabajo, como Inolvidables. Entre los ejemplos más destacados de este último ensamble encontramos a Julie is her name (Liberty, 1955) de Julie London, con Barney Kessel (guitarra) y Ray Leatherwood (bajo); Open Fire, Two Guitars (Columbia, 1959) de Johnny Mathis, con Al Caiola (g), Tony Mottola (g) y Milt Hinton (b); y dos álbumes de Sarah Vaughan, After hours (Roulette, 1961), con Mundell Lowe (g) y George Duvivier (b), y Sarah + Two (Roulette, 1962) con Kessel (g) y Joe Comfort (b). La mayoría de estos músicos son destacados jazzistas y el lenguaje armónico que utilizan en estos discos es innegablemente jazzístico. Sin embargo, la ausencia de batería y el uso infrecuente de tempo swing y solos instrumentales y vocales los han dejado fuera del canon tradicional del jazz. En efecto, estos discos ocupan un espacio liminal entre el jazz y el pop ${ }^{30}$.

Para cantantes como Johnny Mathis y Sarah Vaughan, la reducción en el tamaño del grupo acompañante no fue menor. Mientras que los acompañamientos orquestales requieren de arreglos escritos por profesionales y con antelación, un grupo pequeño puede improvisar en tiempo real, en diálogo con el o la cantante. El productor de Open fire, two guitars, el destacado Mitch Miller, explica que el álbum "fue una idea que desarrollamos ahí mismo en el estudio [...] todo completamente improvisado, sin arreglos"31. Del cantante se espera que colabore de forma mucho más cercana con los músicos. En vez de una estrella que entra al estudio cuando el arreglo ya ha sido realizado y ensayado, se transforma en un músico más. Respecto del proceso de grabación del disco de Vaughan After hours, el guitarrista Mundell Lowe explica: "Sarah nunca nos consideró a nosotros acompañantes y a sí misma

\footnotetext{
29 Stephens 2008: 175.

30 Stephens 2008.

31 Simons 2004: 38-39.
} 
como la estrella; éramos un trío"32. Aunque el repertorio había sido escogido con anticipación, los arreglos fueron concebidos en el estudio y el disco fue grabado en una sesión de solo una noche ${ }^{33}$.

Aunque no sabemos cuánto tiempo tomó grabar Inolvidables, es razonable suponer que la dinámica de trabajo fue similar. La contratapa de las ediciones chilena y brasileña del elepé incluye una fotografía de los músicos en el estudio que refleja muy bien la dinámica sonora del disco. Gatica y Castro aparecen en primer plano, sentados frente a frente con un micrófono Neumann entre ellos. A la derecha y atrás del guitarrista, se ve a Ruiz Pazos y su contrabajo (ver Figura 1).

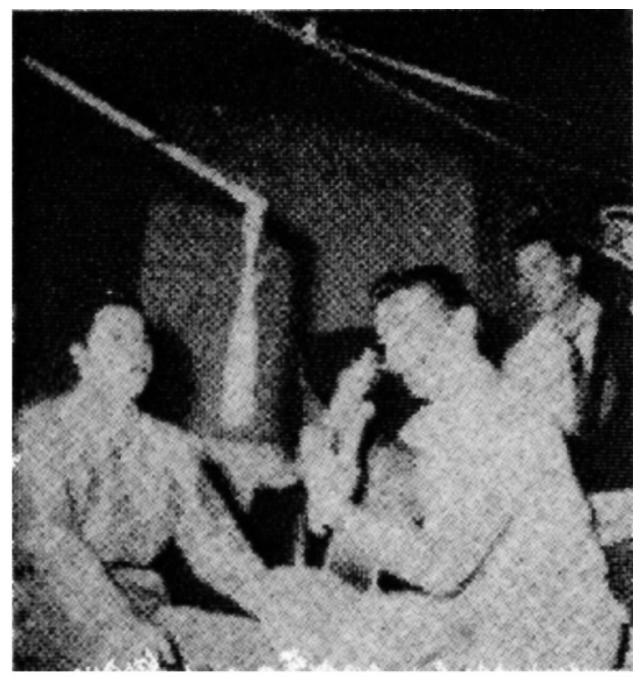

Figura 1: Lucho Gatica, Arturo Castro y Víctor Ruiz Pazos en el estudio de grabación.

En ninguna pista del disco la guitarra se limita a un rol acompañante. En cuanto a volumen, presencia e interés musical, este instrumento se presenta prácticamente al mismo nivel que el cantante. Donde Inolvidables se diferencia de los discos norteamericanos mencionados anteriormente es en el hecho que el contrabajo no tiene un rol de igual importancia al de la guitarra. El bajo se escucha a un volumen considerablemente menor y su función se limita a mantener el pulso y sostener la armonía.

Es importante observar que este ensamble pequeño y delicado de guitarra jazz y contrabajo no es utilizado por crooners interesados en proyectar una imagen masculina dominante y heteronormativa, como las cultivadas por Bing Crosby o Frank

32 Reig y Berger 1990: 100.

33 Reig y Berger 1990: 100. 
Sinatra ${ }^{34}$. Posiblemente esto se deba a que, como hemos visto, el trabajo musical en una agrupación pequeña se presta para la disolución o al menos relajamiento de las jerarquías entre estrellas vocales y músicos acompañantes.

El acompañamiento guitarra-bajo sí era considerado apropiado, y recomendable, para cantantes mujeres, como Julie London y Sarah Vaughan. La asociación con lo femenino es evidente en la manera en que la industria concebía estos discos. Teddy Reig, productor de After hours, le propuso a Vaughan la idea de este disco diciéndole: "En este próximo disco, quiero que cantes desnuda"35. En la contratapa de Julie is her name, el primer disco de London, Bill Balance la presenta así: "Julie es una hermosa, sabrosa, pero sencilla joven con cabello rubio y ojos azules. Estas características son abiertamente evidentes en su afelpado estilo vocal". Es difícil imaginar descripciones de este tipo sobre crooners hombres, como Tony Bennett, Nat King Cole o el mismo Sinatra.

No es sorprendente que el único cantante hombre que grabó un disco con este tipo de ensamble haya sido Johnny Mathis. Como muestra Stephens, Mathis era un croonerque, antes de asumir públicamente su homosexualidad en 1982, cultivó una "sexualidad escurridiza", estrategia que se refleja en sus decisiones musicales, las carátulas de sus discos y en sus entrevistas con la prensa ${ }^{36}$. Lucho Gatica, al igual que Mathis, construyó su estilo incorporando aspectos asociados con genealogías femeninas de performance, como el uso delicado del registro agudo, letras con ambigüedad de género, y repertorios y acompañamientos instrumentales -como los presentes en Inolvidables y Open fire- preferidos por cantantes mujeres ${ }^{37}$. Ambos cantantes lograron que el tema de la preferencia sexual no fuese motivo de acoso por la prensa (al menos a fines de la década del cincuenta), gracias a la mantención de un delicado equilibrio entre características percibidas como femeninas y masculinas. Entre estas últimas podemos incluir las connotaciones del formato elepé descritas anteriormente.

La cara A de Inolvidables con Lucho comienza con "Noche de ronda”, el famoso vals compuesto por Agustín Lara más de dos décadas antes. La versión original ${ }^{38}$ consta de dos partes contrastantes, una primera rapsódica en modo menor ("Noche de ronda/qué triste pasas"), seguida por una sección en tres cuartos y en modo mayor ("Luna que se quiebra/sobre las tinieblas de mi soledad"). Durante los cuarenta y cincuenta, las grabaciones de canción romántica mexicana comienzan, excepto contadas excepciones, con una introducción instrumental. Cualquiera fuese el acompañamiento acordado-guitarras y requinto, piano, big band u orquesta de cuerdas-, lo común era abrir los temas con unos quince a veinte segundos de introducción.

34 Para un fascinante estudio de los crooners y masculinidad ver McCracken 2001.

35 Reig y Berger 1990: 100.

36 Stephens 2010.

37 Gatica modeló su estilo como bolerista en la figura de la cantante cubana Olga Guillot. Para una discusión de la performance de Lucho Gatica y lo femenino ver Party 2012.

38 Grabada en 1937 por Pedro Vargas, junto con la orquesta de Alfonso Esparza Oteo y publicada por RCA Victor. 
En Inolvidables, en cambio, lo que rompe el silencio al comenzar el disco es la voz sola de Gatica, que con un portamento alcanza la nota mi, para cantar, no la primera parte en menor, sino el coro, "Luna que se quiebra" (ver ejemplo musical 1) ${ }^{39}$. Con esa última palabra aparecen el bongó y el contrabajo marcando un pulso predecible. Cuando un momento después aparece la guitarra de Arturo Castro, lo hace de manera sincopada, y desde el primer acorde, de tónica con sexta y novena agregadas, nos transporta al mundo del jazz ${ }^{40}$.

Ejemplo musical 1: "Noche de ronda". Primeros cuatro compases.

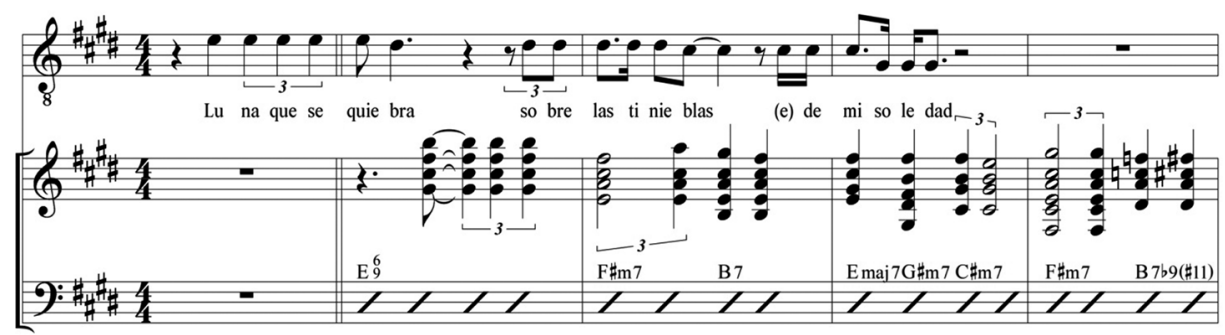

Castro y Ruiz Pazos rearmonizan la canción completa utilizando un lenguaje jazzístico. Si uno compara la armonía original de "Noche de ronda" con la que desarrollan los músicos en este disco es posible distinguir un ritmo armónico más rápido, generalmente de dos acordes por compás, un uso regular de enlaces ii-V-I, típicos del jazz, y acordes extendidos con novenas, oncenas y trecenas, con una amplia gama de alteraciones. El ejemplo musical 2 muestra la armonía de los primeros dieciséis compases del coro ("Luna que se quiebra”) en la versión original y la de Inolvidables. La versión original ha sido transpuesta a mi mayor, para facilitar la comparación.

39 En este artículo el objetivo de las transcripciones musicales es ilustrar, de forma esquemática, recursos melódicos, armónicos y de voicings presentes en el disco. Los ejemplos musicales no pretenden reflejar las sutilezas rítmicas, dinámicas y expresivas presentes en la grabación.

40 La estrategia de comenzar una canción con la voz sin acompañamiento es común en los elepés de crooners con arreglo especial. En Julie is her name se utiliza en 4 de 13 canciones, en Open fire, two guitars, en 2 de 12, y en After hours, en 2 de 11. El abrir el disco solo con la voz, como ocurre en Inolvidables, no tiene precedentes en esta tradición. 
Ejemplo musical 2: "Noche de ronda".

Comparación de la armonía en el original y en la versión de Lucho Gatica.

(Versión original)
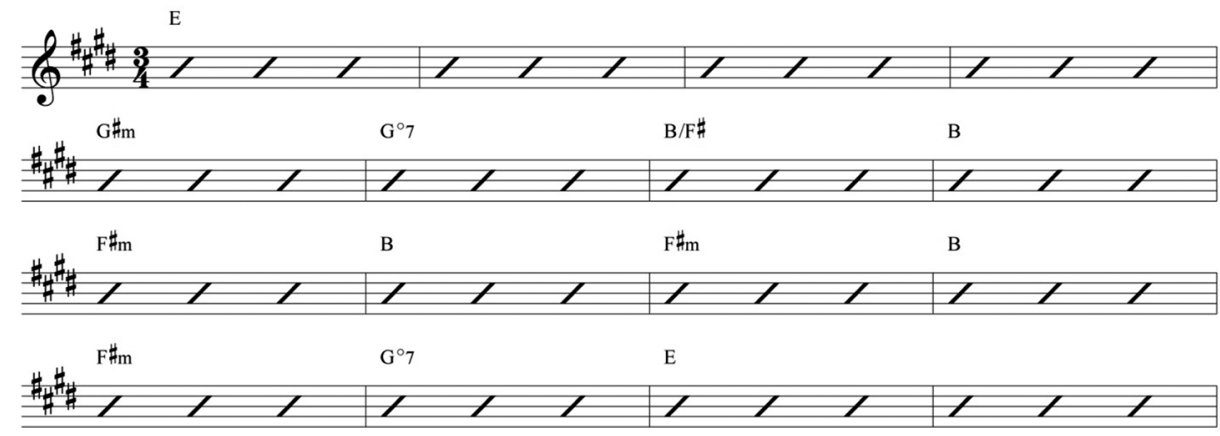

(Versión Inolvidables)

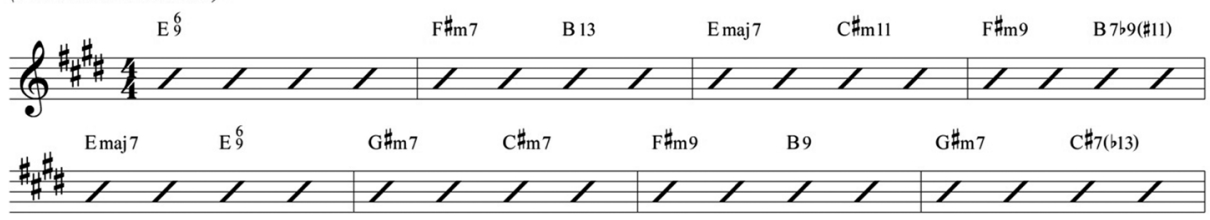

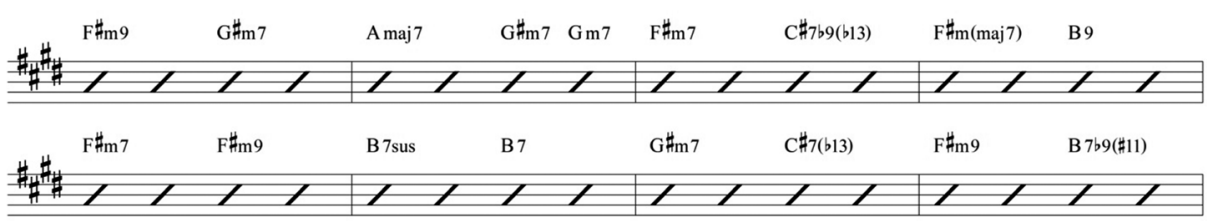

En la versión original, la segunda mitad del coro ("Dile que la quiero / Dile que me muero") comienza con una repetición de la armonía del inicio del coro (tónica, mi mayor). Aquí Gatica canta la melodía original (nota mi, repetida), pero Castro y Ruiz Pazos sustituyen el acorde de tónica por un acorde de subdominante sostenida con séptima menor $(\mathrm{A \# \varpi )}$. Esta sustitución, inusual en la canción romántica mexicana, es un recurso común en el jazz norteamericano para rearmonizar el primer grado de la escala. Un ejemplo de esta práctica ocurre al comienzo de la grabación del standard "I should care" realizada en 1955 por Julie London en el disco anteriormente mencionado, Julie is her name. La composición original, de 1944, armoniza la nota fundamental con un acorde de ii7. La grabación de London, en cambio, comienza con la voz de la cantante entonando la fundamental, mientras que el guitarrista Barney Kessel la acompaña con un iv\#ø arpegiado $^{41}$.

Para ilustrar algunos de los recursos armónico-melódicos preferidos de Arturo Castro en el disco, se incluye la transcripción de una sección más extensa, de la

41 Márquez 2000 presenta un estudio del estilo de Barney Kessel, con un capítulo dedicado al acompañamiento que realiza en el disco Julie is her name. 
segunda parte del coro ("Que vuelva ya”, ver ejemplo musical 3). Entre ellos destacamos el uso del acorde mayor con sexta y novena, la sustitución por tritono (en este caso, Ab7\#11), el uso recurrente de la oncena sostenida, y la supertónica con séptima menor (en este caso, F\#ø). En términos melódicos, Castro complementa las notas tenidas de la voz con pequeños gestos cromáticos en la primera cuerda. Aunque estos no constituyen melodías, en el contexto de un acompañamiento consistentemente acordal, se escuchan como un eco melódico del canto.

Ejemplo musical 3: "Noche de ronda”. Segunda parte del coro.
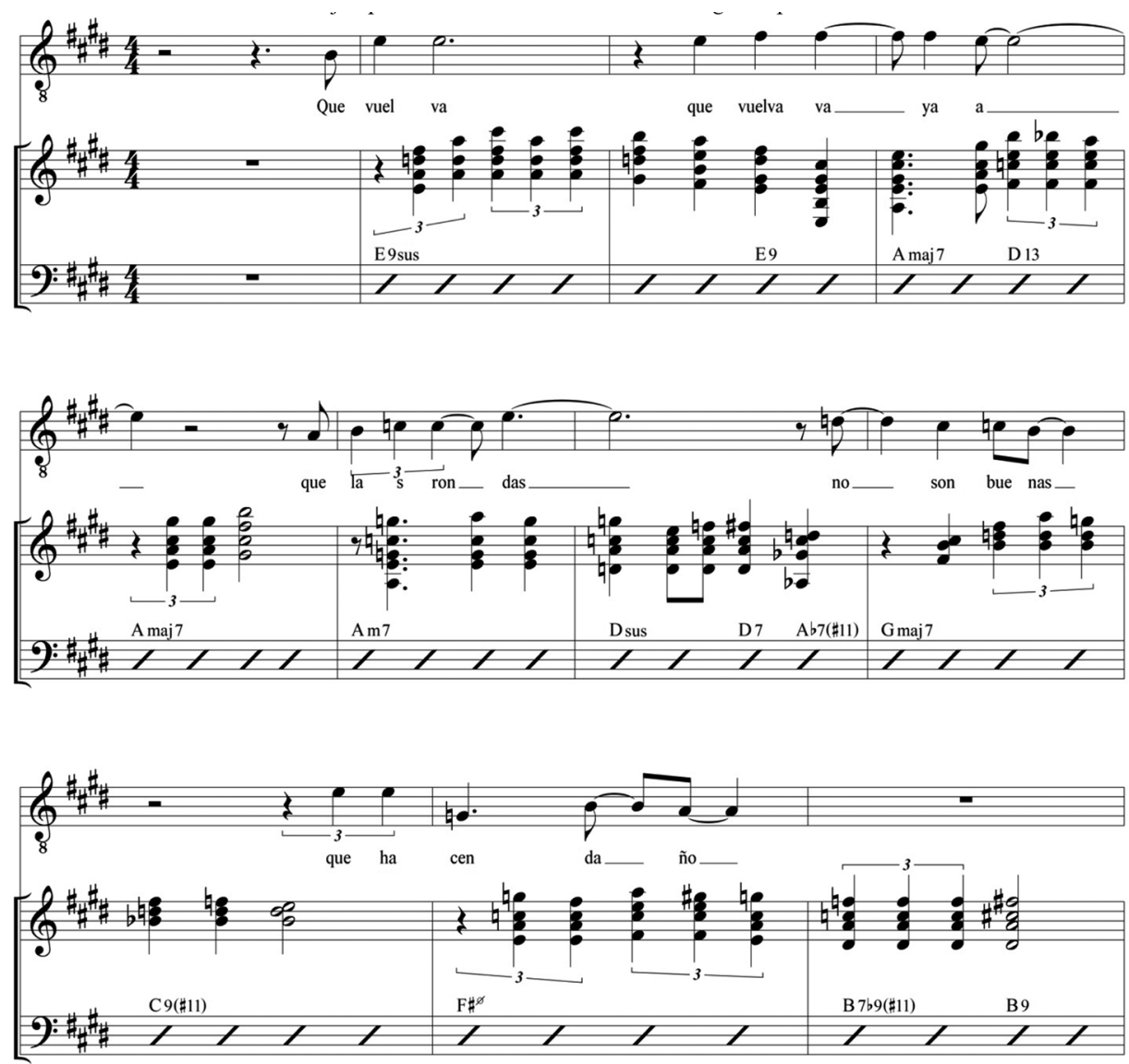

Rearmonizaciones como la de "Noche de ronda" ocurren en todas las canciones del disco. El ejemplo 4 muestra la armonía original de "A la orilla de un palmar" y la propuesta en Inolvidables. En los momentos en que Castro toca solo es donde más libremente explora el espectro armónico jazzístico. En este sentido, es particularmente notable la breve introducción que realiza en la canción de Gabriel Ruiz, "Noche" (ver ejemplo musical 5). 
Ejemplo musical 4. "A la orilla de un palmar". Comparación de la armonía en el original y en la versión de Lucho Gatica.
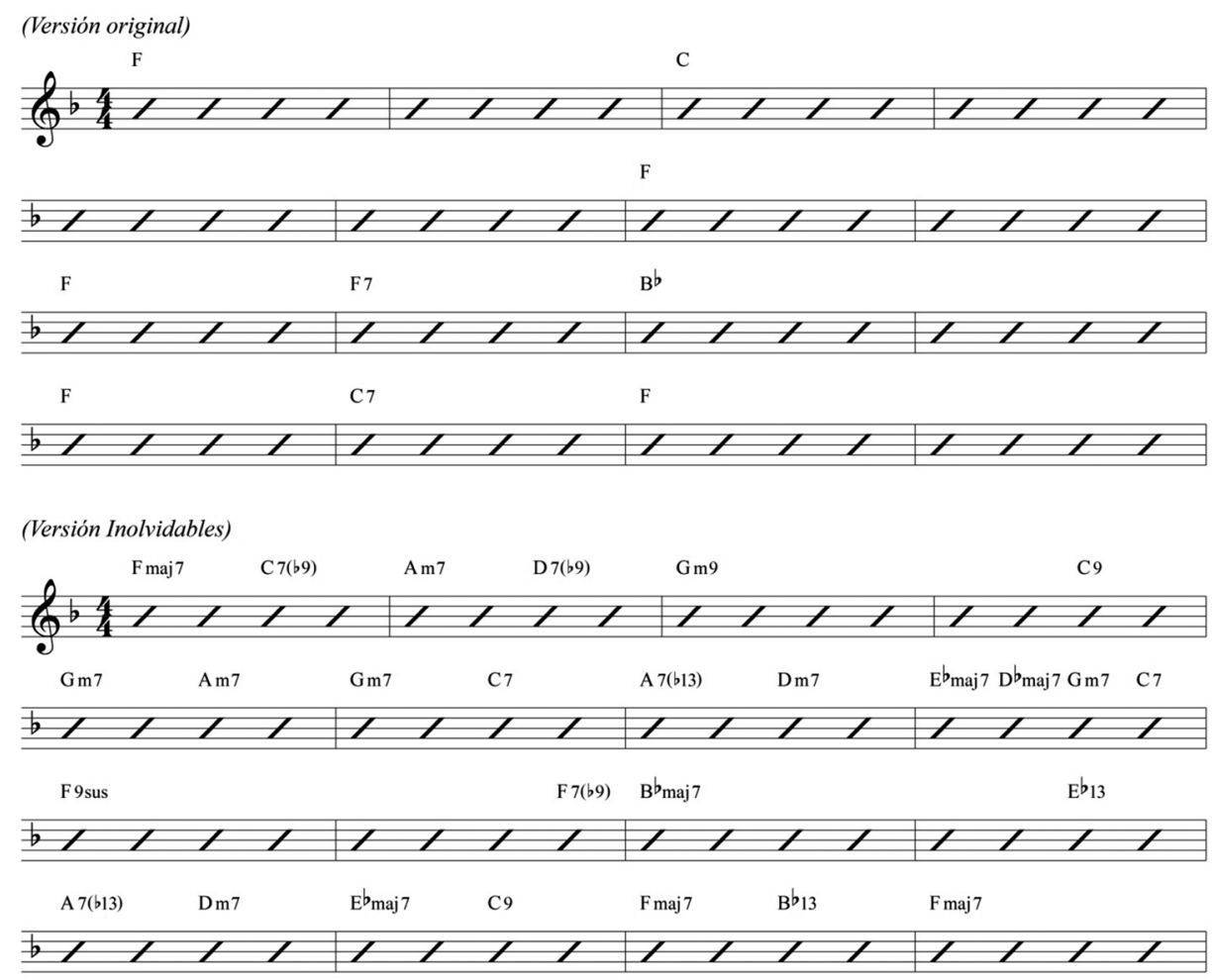

Ejemplo musical 5. "Noche”. Introducción de guitarra sola.

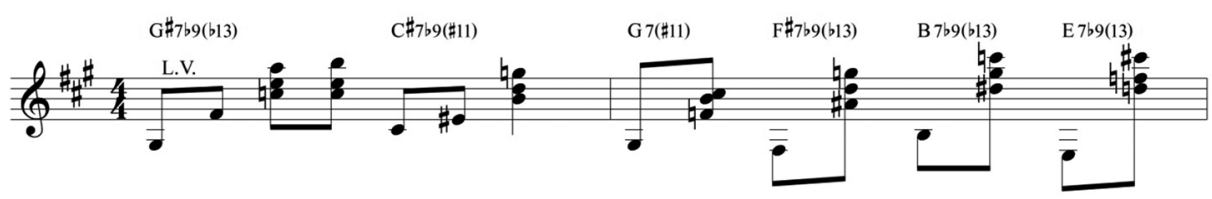

Es posible escuchar a Gatica participando de la rearmonización de los temas en la introducción a "Tú”, el segundo surco del disco. "Tú" es un fox compuesto en 1935 por Gonzalo Curiel (aunque en el registro de derecho de autor fue inscrito a nombre de su hermana, María Elisa). "Tú" consta de 32 compases, estructurados en dos estrofas con forma AA. La grabación más famosa es de Chucho Martínez Gil, quien fuera colaborador directo de Curiel. Lo más probable es que la versión de Martínez Gil haya sido el referente sonoro que Gatica, Castro y Ruiz Pazos utilizaron para grabar esta versión, aunque es posible que también conocieran la de Antonio Badú. 
Una concepción jazzística es reconocible ya en la introducción que los músicos crearon para esta versión. Es una introducción muy breve, de solo dos compases, pero de gran intensidad expresiva. La canción está en sol mayor, y la introducción presenta una vocalización de Gatica respecto de la palabra que da título a la canción, extendiendo la vocal por cuatro blancas, con las notas re-fa-mi-mi bemol. Los músicos acompañan con un acorde por nota cantada de Gatica, con los acordes Bm7 E7b9(\#11) Am7(9) D7(\#9) (ver ejemplo musical 6). Esta progresión, inusual en la canción romántica mexicana, es simple de explicar desde la armonía del jazz. Partiendo de una progresión tradicional de I vi ii V, mediante sustitución diatónica el acorde de tónica es reemplazado por el tercer grado. El sexto grado se sustituye por una dominante del segundo grado, que resuelve al segundo, y termina con la dominante con séptima y novena sostenida -un acorde clásico del jazz-. Particularmente notable es el hecho que la última nota de Gatica (mi bemol) no pertenece al acorde que toca Castro, sino que lo complementa. La melodía que creó Gatica para la introducción no surge de un giro melódico de la canción original, sino de la progresión jazzística que interpretan Castro y Ruiz Pazos.

Ejemplo musical 6. "Tú”. Introducción y primeras dos frases.
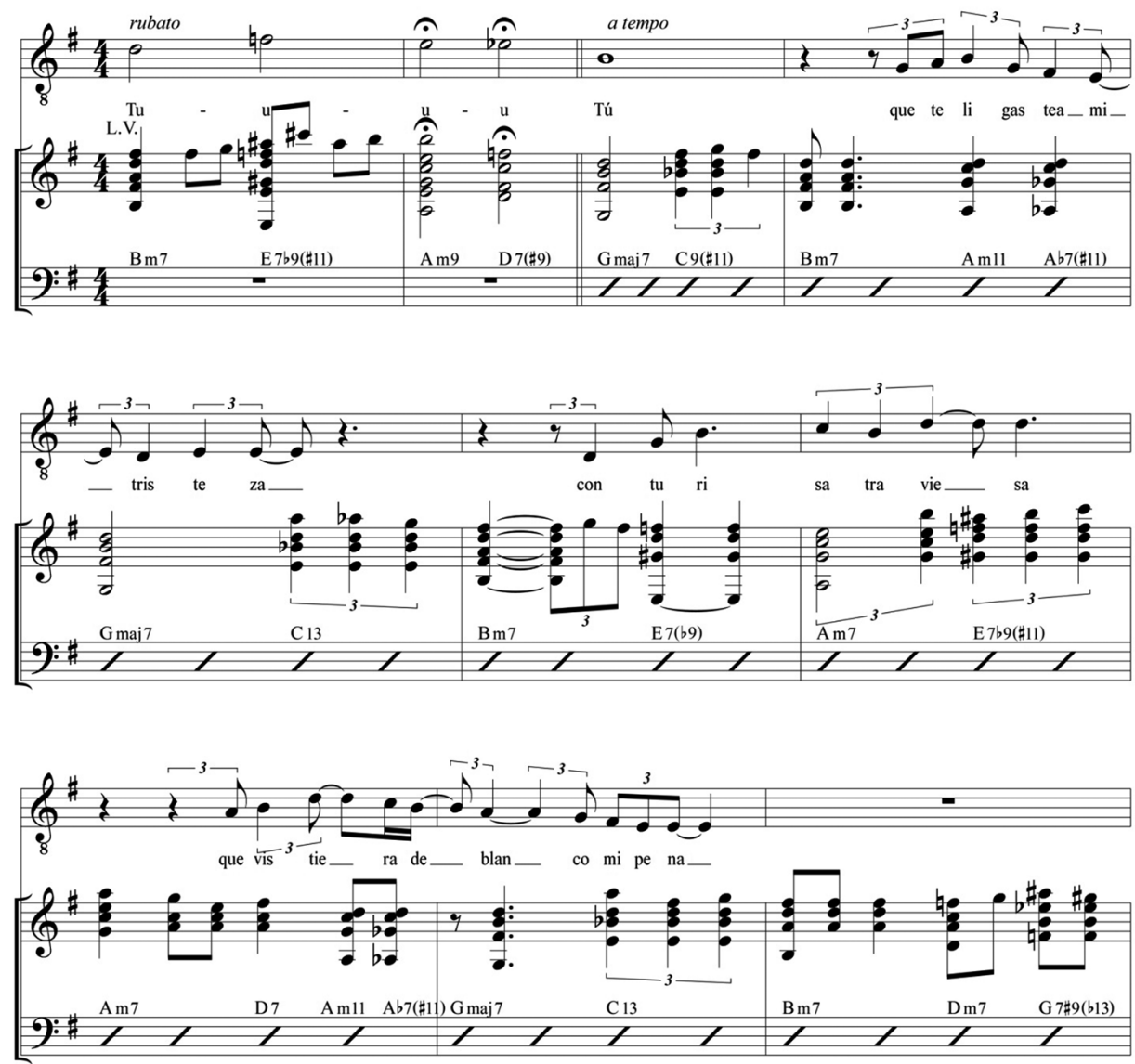


\section{UNA EXPLORACIÓN AL FONDO DE ESTA MÚSICA}

Ciertamente, lo innovador del ensamble y el estilo jazzístico de interpretación presente en Inolvidables con Lucho no pasó inadvertido para Odeón. Los textos que aparecen en las contratapas de las distintas ediciones del álbum muestran tanta confusión como sorpresa. La edición cubana evita mencionar la palabra "jazz", pero sí describe el estilo como "novedoso" y como "una forma nunca antes utilizada por este artista". Arturo Castro y Víctor Ruiz Pazos son mencionados por nombre, y su trabajo se describe como "una labor, no solo difícil, sino de un notable valor artístico”. Pareciera que la sofisticación del acompañamiento complicaba a la disquera, ya que en el párrafo siguiente se insiste en su complejidad, incluso incurriendo en contradicciones: "El acompañamiento, aunque difícil y artístico, es simple".

Nuevamente, la edición chilena (y brasileña, en traducción) nos entrega más y mejor información. "Las versiones aquí contenidas son poco tradicionales", declara, y significan, para Gatica, una "nueva fase", caracterizada por un "modo refinado y exclusivo". Estas últimas características sugieren una concepción del formato elepé que, como vimos anteriormente, sigue la establecida por Capitol Records para Sinatra: un objeto adulto, refinado, serio, permanente y atemporal. Particularmente evocador es el hecho que esta edición reconoce la propuesta del disco como un encuentro entre el jazz norteamericano, el repertorio mexicano y el estilo susurrante de Lucho Gatica:

Con algo elegíaco y conmovedor que tiende sus raíces por todo el corazón de América, desde los litorales del jazz y su nublada "birdland", hasta las sureñas latitudes chilenas que tocan con su telúrica presencia la remota quietud de la Antártida, se ha logrado esa voluntad de comprender y llegar al fondo de esta música para darle todo su significado continental.

Esta descripción también sugiere como un aspecto esencial del disco la voluntad de exploración al fondo de la música, en el sentido de indagar en el contenido musical del repertorio, las frases y giros melódicos, el potencial de la armonía propuesta por la misma melodía. Inevitablemente, la exploración de lo musical se traduce en un cambio en la experiencia de la letra, aunque el foco aquí no es resignificar el contenido semántico de las canciones, como lo logra Chavela Vargas al cantar "Un mundo raro" o la española Martirio en "La bien pagá".

Ciertamente sería posible aventurar una lectura acerca de la resignificación de "Noche de ronda" en la versión del disco. Como mencioné brevemente en la sección anterior, la versión que aparece en Inolvidables comienza no con la primera parte, en menor, sino con la sección más rítmica y memorable, en mayor. Es como si la canción ya hubiese comenzado y nosotros, los auditores, hubiésemos llegado tarde al evento. Pero aún más notable es el hecho que luego de la sección en mayor, Gatica canta la primera parte en menor, en efecto, invirtiendo la estructura de la canción original. El resultado es un vuelco en la dirección narrativa de la canción, que en su versión original parte con el escenario ("el balcón”) para 
pasar por lo que Pérez-Firmat identifica como "verbos de afecto... -herir, lastimar, morir, esperar, hacer daño, dar pena- una secuencia que culmina en llorar, el verbo paradigmático de la poesía sentimental" 42 .

La versión de Gatica, en cambio, abre con la alocución solitaria a la luna y culmina con el cantante contemplando su destino en el balcón, observando a la luna y a su amante en busca de aventuras. Sería correcto afirmar que el culminar en menor le otorga a la canción un carácter más dramático y oscuro. Sin embargo, cambios estructurales como este no se encuentran en el resto del disco. Tampoco otras transformaciones que apunten a reconsiderar el contenido de las letras. Considero que la propuesta estructural de "Noche de ronda" obedece a un deseo de jugar con el material musical, con dar vuelta la forma tradicional de la canción lariana.

En Inolvidables Gatica explora una vocalidad distinta a la que lo hizo famoso a nivel continental. Su voz se adapta al ensamble pequeño e intimista, con un sonido menos englobado, un rango dinámico más reducido, y menos dramatismo en la expresión, en efecto, evitando los gestos grandilocuentes del bolero mexicano de la era dorada. Como en el jazz vocal, en este disco Gatica privilegia la elaboración musical por sobre la comunicación del texto. Más que en cualquiera de sus grabaciones anteriores, en Inolvidables Gatica se aleja del modelo de su mentora, la cubana Olga Guillot. Más que actor o comunicador de un mensaje textual, aquí se escucha a Gatica explorando las posibilidades melódicas y armónicas de cada canción.

El foco en lo musical, y no en la letra, también es relevado por el balance de la grabación entre la voz y la guitarra. A diferencia de la grabación del crooner con orquesta, donde existen dos planos estratificados de manera evidente, aquí el cantante y la guitarra tienen igual relevancia, ambas en tensión con la base rítmica del contrabajo, o contrabajo y bongó. Esto resulta en un disco más exigente para el oyente, ya que la atención del auditor es demandada por más de una fuente.

Otros aspectos también apuntan a la exigencia que el disco presenta para la audiencia. Las catorce canciones son consistentemente breves. Solo dos pasan la marca de los tres minutos, y la mayoría está bajo los dos y medio ${ }^{43}$. Esto significa que hay muy pocas repeticiones, pocas oportunidades de escuchar una vez más ese coro que tanto nos gusta. El aumento en el ritmo armónico y la densidad de los acordes dificultan el saber en qué parte de la canción uno se encuentra. Incluso coros y estrofas se confunden. El hecho que el disco comience sin introducción instrumental alguna nos pone en alerta. La introducción instrumental no solo sirve para establecer la tonalidad del tema, para beneficio del cantante; como la obertura en la música docta europea del siglo XVIII, es una apelación a la audiencia, pide silencio y atención a lo que viene. En Inolvidables los músicos no nos extienden tal invitación.

42 Pérez-Firmat 2008:195.

43 Este aspecto también hace pensar en Julie is her name como un referente. El disco de Gatica contiene 14 canciones en 35 minutos, el de London, 13 en 31 minutos. 
Es como si los músicos, y aquí incluyo intencionadamente al cantante entre los músicos, no estuviesen tocando para nosotros, sino para sí mismos, para su propio placer. El lugar de escucha de Inolvidables no es el teatro elegante del bolero orquestado, ni el salón de baile de las big bands, ni el espacio público de la serenata de los tríos románticos, ni el cabaré con piano de Agustín Lara. Inolvidables es un disco intimista, no en el sentido tradicional de generar la fantasía de estar a solas con el crooner $^{44}$, sino de un encuentro privado entre músicos. No es difícil imaginar este encuentro como se ve en la imagen de la contratapa: tres músicos en torno a un solo micrófono. Todo esto sugiere un carácter informal, improvisado. Nuevamente, no en el sentido tradicional de improvisación en jazz -vía solos instrumentales- sino de decisiones musicales tomadas en el momento.

\section{EL IMPACTO: INOLVIDABLESY LA BOSSA NOVA}

Debido a lo exigente del disco, no es sorprendente que Inolvidables no tuviese un importante impacto a nivel comercial ni estilístico en la carrera de Gatica. De hecho, no se convirtió en la "nueva fase" que prometían las carátulas, sino que quedó como un experimento aislado en la discografía del cantante. En cambio, el disco sí tuvo acogida entre músicos de la región, lo que se refleja en grabaciones similares en cuanto a ensamble y estilo interpretativo. Entre las más destacadas están las grabaciones que realizó José Feliciano en 1966 para RCA en Argentina, de boleros acompañados de guitarra, contrabajo y percusión, y Pablo Milanés a partir del segundo volumen de su serie de álbumes titulados Filin (1989 en adelante).

Es posible que Feliciano no conociese el disco Inolvidables al momento de decidir incorporar elementos de jazz y blues en sus grabaciones en Argentina, pero en el caso de Milanés esto es menos probable. En sus comienzos, a mediados de los años cincuenta, Milanés no solo interpretaba canciones del repertorio de Gatica, sino que modeló su estilo temprano en el cantante chileno. De hecho, sus contemporáneos consideraban que "cantaba imitando mucho a Lucho Gatica"45. De la serie Filin, particularmente cercano a Inolvidables es el volumen 4, de 1991, ya que su repertorio también se encuentra acotado a canciones mexicanas. El disco comparte cuatro canciones con Inolvidables, "Noche de ronda", "La borrachita", "Verdad amarga" y "Nocturnal". Aunque Milanés no ha reconocido haber sido influenciado por Inolvidables, llama la atención que su versión de "Noche de ronda" utiliza la misma estructura invertida -altamente inusual, de comenzar en mayor y culminar en menor- que aparece en el disco de Gatica.

Pero quizá lo más sorprendente es el impacto que Inolvidables con Lucho tuvo en Brasil, entre los compositores-guitarristas que crearon la bossa nova. Roberto Menescal y Carlos Lyra, dos artistas centrales en los orígenes de la bossa nova, han reconocido que Inolvidables fue fundamental para su desarrollo. Basada en

44 González 2000.

45 Díaz 2001: 25. 
una entrevista con Menescal realizada en 1998, la investigadora Santuza Cambraia Naves escribe,

Roberto Menescal, por ejemplo, al hablar sobre las novedades extranjeras que lo marcaron profundamente durante su periodo de formación musical, se refiere al elepé Inolvidable [sic] de Lucho Gatica, que en su arreglo recurría solo a dos instrumentos, guitarra y bajo, rompiendo con la tradición del bolero de utilizar grandes orquestaciones. Según Menescal, este procedimiento fue importante para que él y otros músicos de su generación desarrollaran el hábito de oír la guitarra, que así, dialogando apenas con el bajo, aparece destacada, singularizada. ${ }^{46}$

Carlos Lyra es aún más explícito al considerar, en su autobiografía, a Inolvidables como un precursor de la bossa nova:

Entre aquellos que considero precursores de la Bossa Nova [...] tengo un lugar reservado en el corazón para el bolero mexicano de compositores como Agustín Lara, Gonzalo Curiel y Maria Griver [sic], sobre todo en la interpretación de Lucho Gatica, quien, en los años cincuenta, lanzó un disco de boleros: Inolvidables, acompañado por una guitarra sorprendente de Arturo Castro, que removió las cabezas de los muchos que amábamos el instrumento a ese género de música. ${ }^{47}$

Las declaraciones de Menescal y Lyra son inusuales por el hecho de reconocer un referente hispanoamericano para el desarrollo de la guitarra de la bossa nova, particularmente un precursor asociado con el bolero mexicano. Como diversos autores han argumentado, el discurso de validación de la bossa nova estuvo marcado notablemente por una posición antibolero y antimexicana ${ }^{48}$. Naves muestra que ya en 1968 los defensores de la bossa nova "son unánimes en atribuir a João Gilberto una postura que valoriza la contención, contraria a la emotividad excesiva de la música popular de las décadas de los cuarenta y cincuenta"49. Según estos autores, "los músicos de la bossa nova, especialmente João Gilberto, pautarían su trabajo a través del rechazo a las sambas-canções y a los boleros melodramáticos del período anterior" 50 .

Simone Luci Pereira concuerda, y propone que, el bolero, "con sus aspectos subjetivos, imprevistos, no lineales, dionisíacos, aparece opuesto a algo más racional, apolíneo, lineal, organizado y, por sobre todo, legitimado, como la bossa nova o la MPB" 51 . El bolero hispanoamericano es presentado como de menor calidad y sofisticación que la bossa nova. "En Brasil", sugiere Pereira, "el bolero es muchas veces escuchado, pensado y hegemónicamente construido por la historiografía musical como inferior a la bossa nova, estilo o género que fue su contemporáneo

46 Naves 2000: 36. Traducción del autor.

47 Lyra 2008: 34. Traducción del autor.

48 Araújo 1999; Naves 2000; Duarte 2008; Ulhoa 2010; Pereira 2016; Alonso 2016.

49 Naves 2000: 35.

50 Naves 2000: 35.

51 Pereira 2016: 148. Traducción del autor. 
en la historia de la música popular brasileña de los años 1950/60, aunque goce de amplia aceptación popular y haya sido incorporado en la música brasileña más popularmente difundida"52.

En el conocido estudio de bossa nova de Ruy Castro, el referente que sí aparece de manera consistente como influencia para su desarrollo es el guitarrista norteamericano de jazz Barney Kessel, en particular su participación como acompañante en el elepé Julie is her name (1955), de Julie London ${ }^{53}$. Lo notable de los comentarios de Menescal y Lyra es que reconocen un paso intermedio entre la guitarra jazz norteamericana y la guitarra bossa, y este sería la labor de Arturo Castro en Inolvidables con Lucho. Sugieren que Inolvidables les mostró que era posible adaptar el estilo jazzístico de Barney Kessel, interpretado con una Gibson ES-350 con cuerdas de metal, al acompañamiento de un repertorio latinoamericano con una guitarra con cuerdas de nylon. Como destaca Lyra, "La guitarra de Arturo Castro fue única en el mundo. Tan importante como la de Barney Kessel. Es la guitarra más bonita que he oído en mi vida" 54 .

A sesenta años de su lanzamiento, no sería correcto afirmar que el elepé Inolvidables con Lucho es un disco desconocido. Generalmente aparece listado en biografías y discografías de Lucho Gatica y, aunque bajo otros nombres, es posible encontrarlo en plataformas de descarga digital o de streaming 55 . Sin embargo, en la bibliografía existente se le menciona como un elepé antológico más -probablemente un resultado de la ambigüedad de su título-.

El objetivo de este artículo ha sido demostrar las múltiples maneras en que Inolvidables dista de ser un elepé más. Inolvidables demuestra un conocimiento acabado de la innovadora propuesta de Capitol Records, en su trabajo con Frank Sinatra, de concebir un elepé como un concepto unificado. Al igual que en los discos de Sinatra con Capitol, de mediados de la década del cincuenta, Inolvidables privilegia un repertorio de canciones antiguas, un acompañamiento instrumental homogéneo y un carácter consistente en las dos caras del disco. Todas estas decisiones se traducen en que Inolvidables con Lucho sea un elepé unificado, uno de los primeros en América Latina ${ }^{56}$. Pero igualmente importante es el hecho que concebir un elepé de esta manera implicaba muchas connotaciones asociadas al formato y al Sinatra de este periodo, connotaciones de seriedad, adultez, masculinidad y refinamiento.

52 Pereira 2016:148.

53 Castro 1990.

54 Ordóñez 2006.

55 En 2018, el disco está disponible en Spotify, Apple Music y Amazon.com bajo el nombre de The Music of Chile/Lucho Gatica, a Bolero Singer (Black Round Records, 2009) y en la antología Lucho Gatica, vol. II (Orfeon 2011).

56 Paiano sugiere que el primer elepé unificado de música brasilera fue el disco fundacional de la bossa nova, Chega de Saudade, de João Gilberto (citado en Stroud 2008: 55). Chega de Saudade fue lanzado meses después que Inolvidables, por el mismo sello discográfico (Odeón). 
Donde Inolvidables difiere de manera fundamental del referente Sinatra es en la elección del ensamble que acompaña al cantante. Un grupo pequeño en torno a una guitarra con cuerdas de nylon, interpretando con maestría en un estilo de jazz moderno, sugiere un modelo radicalmente distinto. Aquí el referente clave pareciera ser el elepé Julie is her name, de la actriz y cantante Julie London. De este modo, Gatica confirma su interés en adoptar prácticas performáticas de artistas mujeres -temprano en su carrera su modelo había sido el estilo de Olga Guillot-. Los puntos de encuentro entre el disco de London y el de Gatica se evidencian en la recepción e impacto que ambos elepés tuvieron entre los músicos brasileños que desarrollaron la bossa nova. Para ellos, los elepés de London y Gatica fueron igualmente influyentes en su proyecto de renovar el acompañamiento de canciones populares mediante el lenguaje de la guitarra jazz.

\section{BIBLIOGRAFÍA}

Acosta, LEONARDO

1988 "El Bolero y el kitsch”. Letras Cubanas, 9, pp. 58-76.

Alonso, Gustavo

2016 "Influencia mexicana en la música brasileña", Historia Social De Las Músicas Populares Latinoamericanas: Una Visión Desde México. Editado por Luis Omar Montoya Arias y Gabriel Medrano de Luna. Guanajuato: Universidad de Guanajuato, 2016, pp. 9-47.

Araújo, Samuel

1999 "The Politics of Passion: The Impact of Bolero on Brazilian Musical Expressions", Yearbook for Traditional Music, 31, pp. 42-56.

Castro, RuY

1990 Chega De Saudade: A História E as Histórias Da Bossa Nova. Companhia das letras.

Contreras, Félix

1989 Porque Tienen Filin. Santiago de Cuba: Editorial Oriente.

Díaz Pérez, Clara

2001 Pablo Milanés: Con luz propia. Navarra: Editorial Txalaparta.

Duarte Valente, Heló́sa de Araújo

2008 "The Blurred Soft Beat of Smouldering Hearts: The Nomadic Bolero", Lied und populäre Kultur/Song and Popular Culture, 53, pp. 95-108.

García Yero, Cary Aileen

2012 "Is it Just About Love?: Filin and Politics in Prerevolutionary Cuba", Studies in Latin American Popular Culture, 30/1, pp. 138-161.

Gilbert, Roger

1998 "The Swinger and the Loser: Sinatra, Masculinity, and Fifties Culture", Frank Sinatra and Popular Culture: Essays on an American Icon. Editado por Leonard Mustazza. Praeger Publishers, pp. 38-49.

Giro, RADAMÉS

2001 El Filin de César Portillo De La Luz. La Habana: Ediciones Unión. 
GonzÁlez, Juan Pablo

2000 "El canto mediatizado: Breve historia de la llegada del cantante a nuestra casa", Revista Musical Chilena, LIV/194, pp. 26-40

Keightley, KeIr

2001 "You Keep Coming Back Like a Song: Adult Audiences, Taste Panics, and the Idea of the Standard", Journal of Popular Music Studies, 13/1, pp. 7-40.

2004 "Long Play: Adult-Oriented Popular Music and the Temporal Logics of the Post-War Sound Recording Industry in the USA", Media, Culture E' Society, 26/3, pp. 375-391.

2008 "Music for Middlebrows: Defining the Easy Listening Era, 1946-1966", American Music, 26/3, pp. 309-335.

LyRa, CARLOS

2008 Eu e a bossa: Uma História Da Bossa Nova. Casa da Palavra.

Márouez, Esteban

2000 “Incorporating Barney Kessel's Methodology in Jazz Guitar Instruction”. Tesis. The University of Texas at El Paso.

Márquez, Pablo

2013 El rey Lucho cantaba boleros. Santiago: Aguilar.

McCracken, Allison

2001 "Real Men Don't Sing Ballads: The Radio Crooner in Hollywood, 1929-1933", Soundtrack Available: Essays on Film and Popular Music. Editado por Pamela Robertson Wojcik y Arthur Knight. Durham: Duke University Press, pp. 105-133.

Monsiváis, Carlos

1984 "La agonía interminable de la canción romántica", Comunicación y Cultura, 12, pp. 21-39.

Naves, Santuza Cambraia

2000 "Da bossa nova à Tropicália: contenção e excesso na música popular", Revista Brasileira de Ciências Sociais, 15/43, pp. 35-44.

ORDÓÑEZ, ANDRÉS

2006 "Memorias De Un Brasileño", La Jornada Semanal. Disponible en www.jornada. unam.mx/2006/10/08/sem-andres.html

OSBORNE, RichARD

2012 Vinyl: A History of the Analogue Record. New York: Routledge.

Party, Daniel

2012 “'Un pequeño defecto’: El bolero de Lucho Gatica entre sus fans y la crítica”, $E l$ lenguaje de las emociones: Afecto y cultura en América Latina. Editado por Mabel Moraña e Ignacio Sánchez Prado. Madrid: Iberoamericana/Vervuert, pp. 227-242.

Pereira, Simone Luci

2016 “¿Qué Bolero?” - Questões Sobre Narrativas, Escutas, Migrações, Identidades”, Canção Romântica: Intimidade, Mediação E Identidade Na América Latina. Editado por Martha Ulhôa y Simone Luci Pereira. Rio de Janeiro, Brasil: Letra e Imagem Editora, pp. 145-169.

Pérez Firmat, Gustavo

2008 "Latunes: An Introduction”, Latin American Research Review, 43/2, pp. 180-203. 
Poe, Karen

1996 Boleros. Heredia, Costa Rica: Editorial de la Universidad Nacional.

Reig, Teddy y EdWARd Berger

1990 Reminiscing in Tempo: The Life and Times of a Jazz Hustler, Metuchen, New Jersey: The Scarecrow Press and the Institute of Jazz Studies.

Rojas Donoso, Gonzalo

1992 Contigo en la distancia: Lucho Gatica, el rey del bolero. Santiago: Ediciones Cerro Huelén.

SAAVEDRA, LEONORA

2009 "Manuel M. Ponce's Chapultepec and the Conflicted Representations of a Contested Space”, The Musical Quarterly, 92/3-4, pp. 279-328.

Simons, Dave

2004 Studio Stories: How the Great New York Records Were Made: From Miles to Madonna, Sinatra to the Ramones. San Francisco, Calif.: Hal Leonard Corporation.

STEPHENS, VINCENT

2008 "Crooning on the Fault Lines: Theorizing Jazz and Pop Vocal Singing Discourse in the Rock Era, 1955-1978", American Music, 26/2, pp. 156-195.

2010 "Shaking the Closet: Analyzing Johnny Mathis's Sexual Elusiveness, 1956-82", Popular Music and Society, 33/5, pp. 597-623.

STROUd, SEAN

2008 The Defence of Tradition in Brazilian Popular Music: Politics, Culture and the Creation of Música Popular Brasileira. Burlington, VT: Ashgate Publishing.

Ulhôa, Martha Tupinambá de

2010 "Bolero, Bossa Nova y Fílin: Estética e ideología en la música brasileña”, III Congreso Internacional Música, Identidad Y Cultura En El Caribe - "el bolero en la cultura caribeña y su proyección universal”. Editado por Darío Tejeda y Rafael Emilio Yunén. Santiago de los Caballeros: Instituto de Estudios Caribeños, Centro León, Ministerio de la Cultura, pp. 515-522.

VARgas Llosa, Mario

1977 La tía Julia y el escribidor. Barcelona: Seix Barral.

\section{REVISTAS}

Long Playing: Revista Geral de Discos Fabricados no Brasil, III/16 (enero-febrero, 1959). 